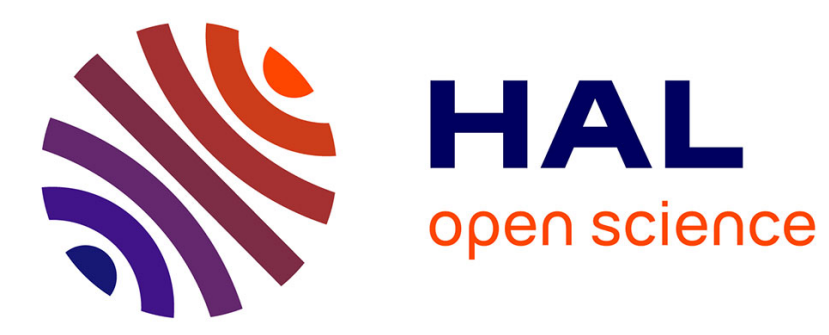

\title{
Links Between Steepened Mach Waves and Coherent Structures for a Supersonic Jet \\ Pierre Pineau, Christophe Bogey
}

\section{To cite this version:}

Pierre Pineau, Christophe Bogey. Links Between Steepened Mach Waves and Coherent Structures for a Supersonic Jet. AIAA Journal, 2021, pp.1 - 9. 10.2514/1.j059859 . hal-03210322

\section{HAL Id: hal-03210322 \\ https://hal.science/hal-03210322}

Submitted on 28 Apr 2021

HAL is a multi-disciplinary open access archive for the deposit and dissemination of scientific research documents, whether they are published or not. The documents may come from teaching and research institutions in France or abroad, or from public or private research centers.
L'archive ouverte pluridisciplinaire HAL, est destinée au dépôt et à la diffusion de documents scientifiques de niveau recherche, publiés ou non, émanant des établissements d'enseignement et de recherche français ou étrangers, des laboratoires publics ou privés. 


\title{
Links between steepened Mach waves and coherent structures for a supersonic jet
}

\author{
Pierre Pineau * and Christophe Bogey ${ }^{\dagger}$ \\ Univ Lyon, École Centrale de Lyon, INSA Lyon, Université Claude Bernard Lyon I, CNRS, \\ Laboratoire de Mécanique des Fluides et d'Acoustique, UMR 5509, F-69134, Ecully, France
}

The links between coherent structures in the shear-layer of an isothermal Mach 2 jet and steepened Mach waves just outside the jet are investigated using large-eddy simulation. Conditional averages triggered by the detection of high-intensity pressure peaks on a conical surface near the jet flow are computed. Averages obtained for peaks of different intensities are presented to identify the factors favoring the steepened aspect of the waves, unlikely due to non-linear propagation effects given the surface radial position. The Mach waves just outside the jet are shown to be correlated with coherent structures in the supersonic side of the mixing layers. Their steepened aspect is found to increase with their intensity. The variations of the wave properties with the convection speed, strength and geometrical shape of the mixing layer structures are then discussed. These properties do not depend much on the convection speed, but are closely related to the two other parameters. The wave intensity and steepened aspect increase as the shear-layer structures are stronger, but also as they are more inclined relative to the jet direction. In the latter case, the asymmetric shape of the structure appears to promote the formation of positively skewed Mach waves.

\section{Nomenclature}

$$
\begin{aligned}
T & =\text { temperature } \\
p & =\text { pressure } \\
\rho & =\text { density } \\
u & =\text { velocity } \\
a & =\text { speed of sound } \\
M & =\text { Mach number } \\
v & =\text { kinematic viscosity } \\
\gamma & =\text { ratio of specific heats }
\end{aligned}
$$

\footnotetext{
*Post-Doctoral fellow, pierre.pineau@ec-lyon.fr

${ }^{\dagger}$ CNRS Research Scientist, AIAA senior member

Preliminary version of the results presented as Paper 2018-3305 at the 2018 AIAA/CEAS Aeroacoustics Conference, Atlanta, GA, June 25-29
} 


$$
\begin{array}{ll}
r_{s} & =\text { specific ideal gas constant } \\
r & =\text { radial coordinate } \\
\theta & =\text { azimuthal coordinate } \\
z & =\text { axial coordinate } \\
t & =\text { time } \\
\Delta r & =\text { radial mesh spacing } \\
\Delta \theta & =\text { azimuthal resolution } \\
\Delta z & =\text { axial mesh spacing } \\
D & =\text { jet diameter } \\
r_{0} & =\text { jet radius } \\
R_{D} & =\text { diameter-based Reynolds number } \\
\alpha & =\text { Mach wave radiation angle } \\
\tau & =\text { interval length for the conditional averages }
\end{array}
$$

\section{Subscripts}

$$
\begin{aligned}
& \infty \quad=\text { ambient quantity } \\
& j \quad=\text { initial value of the jet parameters } \\
& \text { trig } \quad=\text { conditional averages trigger } \\
& s \quad=\text { stagnation quantity }
\end{aligned}
$$

Superscripts

= $\quad$ fluctuating quantity

\section{Introduction}

High-speed supersonic jets such as those exiting from the jet engines powering modern military aircrafts produce a distinctive, particularly unpleasant sound known as crackle [1] which has been related to the presence of steep, jagged shock structures in their acoustic fields. These steepened waves primarily occur in the downstream direction, where acoustic levels are strongest, and have been reported near jets issued from model scale nozzles [2-5] as well as full-scale engines [1, 6, 7]. Several nonlinear mechanisms are involved in the formation of these waves. Notably, nonlinear propagation effects due to high pressure levels in the acoustic field can cause a gradual steepening of the waves as they propagate away from the flow, leading to the formation of shocks, as observed, for instance, by Gee et al. [7] for full-scale jet engines. Another consequence of nonlinear propagation effects is the coalescence of neighbouring waves, which leads to fewer and stronger shocks as the propagation distance increases [9, 10]. As argued by Lighthill [11], this mechanism promotes the formation of extreme, intermittent, steepened waves such as those responsible for the 
perception of crackle. For a given broadband signal, the propagation distance required for shock formation can be estimated based on the initial wave amplitude and peak frequency [12]. Based on the model of Baars et al. [13] for spherically-spreading waves, this distance ranges from just a few diameters for full-scale jet engines to several hundred for laboratory-scale tests, which is beyond the typical propagation distances considered in anechoic chambers. Moreover, for small-scale nozzles, the peak frequencies are higher than for full-scale nozzle so that the effects of molecular absorption, which counteract shock formation, are stronger. It is thus likely that nonlinear propagation effects are not the only mechanism at play in the formation of crackle. This has led some authors to argue that shock formation also occurs at the source, inside the turbulent flow, which has been confirmed by optical visualizations [5, 14, 15], as well as numerical simulations [16-18] of jets and mixing layers showing the presence of shocklets embedded inside the turbulent flow and propagating in the far field.

Despite several years of research, this source steepening mechanism is still not fully understood. For highly supersonic jets, the dominant noise component in the downstream direction, where crackle perception is most intense, consists of Mach waves generated by coherent structures [19, 20]. These structures are convected at a supersonic speed $u_{c}$ inside the jet flow and lead to a peak of noise emission for an angle $\alpha$ given by

$$
\cos \alpha=\frac{a_{\infty}}{u_{c}}
$$

with respect to the jet direction, where $a_{\infty}$ is the speed of sound in the ambient medium. Since coherent structures act upon the ambient medium as wavy walls or bluff bodies, the formation of shocks is not surprising as soon as the convection velocity exceeds the speed of sound. However, in most models of Mach wave radiation, coherent structures and their associated pressure fields are modelled as linear instability waves [21--24]. These models correctly predict several important features of Mach wave radiation, such as the direction of maximum noise level or the shape of the spectra near the peak frequency but fail to take into account the possible presence of shocks, due to their linear nature. Therefore, further investigations are required in order to characterize the formation mechanism of steepened Mach waves, and to highlight what particular aspects of the source, that is, the coherent structures, causes the wavefronts to steepen near the jet. This is particularly important since previous studies have shown that modifying these structures using noise reduction devices such as chevrons [25], microjets [26] or nozzle inserts [27] could reduce the crackling behaviour of supersonic jets.

Recently, by combining simulations of temporally-developing plane mixing layers and a parametric analysis of supersonic flows over wavy surfaces, Buchta \& Freund [17, 28] related the skewness of the near pressure field, hence its nonlinearity, to the convection speed and the magnitude of the turbulent fluctuations. Similar results were obtained by the authors of the present paper using numerical simulations of temporally-developing axisymmetric mixing layers [29-31]. In the latter studies, the coherent structures generating the steepened waves were extracted by averaging a large number 
of flow events correlated with intense pressure peaks near the jet flow. The shape and convection speed of the structures were both found to influence the intensity and the nonlinearity of the waves they generate. It is noteworthy that the results obtained in the aforementioned studies are based on the analysis of temporally-developing model flows. These models are useful for performing parametric studies revealing the noise generation mechanisms at play. However, since they remain parallel as they grow, they differ from the spatially-developing jets considered in experiments which spread with increasing distance from the nozzle. For that reason, coherent structures in spatially-developing jets are expected to have different geometrical properties [32] and convection speeds [33] than those in temporally-developing ones so that it is legitimate to wonder how this would affect the steepened Mach waves they generate.

In the present study, a conditional averaging procedure very similar to the one described in Refs. [29,-31, 34] for temporally-developing jets is applied to a numerical simulation of an isothermal Mach 2 jet in order to investigate the role of large-scale coherent structures in the steepening of Mach waves immediately inside the turbulent flow. The conditional-averaging procedure is based on the detection and synchronization of extreme pressure peaks on a conical surface spreading with the jet. This surface is located as close as possible to the jet shear layer to minimize the wave steepening due to nonlinear propagation effects. By sorting the detected peaks according to their peak intensity, the structures generating the strongest waves can be compared with those that produce the weakest ones in order to identify which ones of their properties favor the steepening of Mach waves. More particularly, based on previous investigations [27-29, 35], the roles of the structure convection speeds, strengths and geometrical shapes will be explored. Unlike the temporally-developing flows considered in Refs. [29-31], the present jet exhausts from a nozzle and spreads with the axial coordinate, so that the structure tendency to generate steepened Mach waves is expected to evolve with the distance from the nozzle. For that reason, the conditional-averaging procedure will be performed for different axial positions spanning the entire axial extent of the shear layer.

The paper is organized as follows. First, the simulation parameters and computational methods are introduced in section [II Then, the conditional averaging procedure is presented and applied to the numerical database in section III] Finally, concluding remarks are given in section IV.

\section{Simulation parameters}

\section{A. Jet parameters}

The jet considered in the present study is isothermal and exhausts from a round, straight-pipe nozzle at a static pressure $p_{j}$ matching the ambient pressure $p_{\infty}=10^{5} \mathrm{~Pa}$. It has an exit Mach number $M_{j}=u_{j} / \sqrt{\gamma r_{s} T_{j}}$ of 2 , where $u_{j}$ is the exit velocity, $T_{j}$ is the exit static temperature equal to the ambient temperature $T_{\infty}=293 \mathrm{~K}, \gamma=c_{p} / c_{v}=1.4$ is the ratio of specific heat and $r_{s}$ is the specific ideal gas constant. The total temperature of the jet is equal to $T_{s}=1.8 T_{\infty}$ and the diameter-based Reynolds number is equal to $R e_{D}=u_{j} D / v_{j}=5 \times 10^{4}$, where $D=2 r_{0}$ is the jet diameter and 

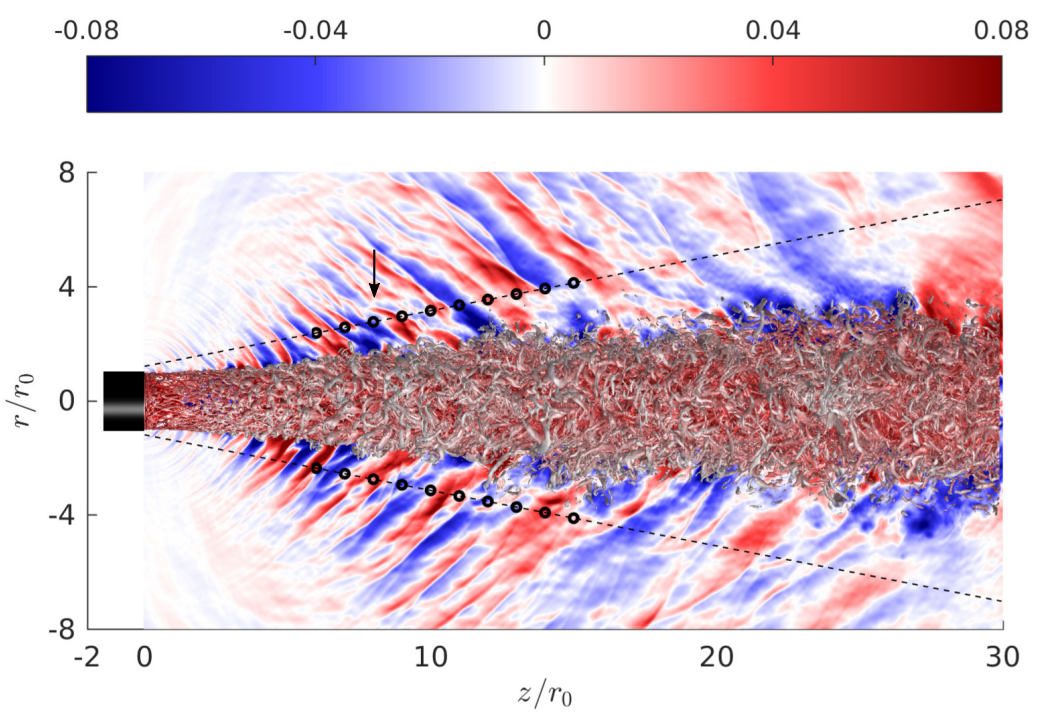

Fig. 1 Positive isosurface of q-criterion $q=0.65\left(u_{j} / r_{0}\right)^{2}$ colored by the jet velocity and pressure fluctuations $p^{\prime} / p_{\infty}$ outside the jet. The circles represent the detection positions for the conditional averages and the arrow indicates the location of peak pressure levels on the cylindrical surface.

$v_{j}$ is the kinematic viscosity at the nozzle exit, computed from the Sutherland's law. Inside the nozzle, a Blasius-like velocity profile with a thickness of $0.076 r_{0}$ is prescribed. This value yields a shear-layer momentum thickness of $\delta_{\theta} / r_{0}=2 / \sqrt{R e_{D}}=8.9 \times 10^{-3}$ at the nozzle exit, following an empirical law proposed by Zaman [36] for initially laminar jets. In order to favor the transition of the shear layers from a laminar to a turbulent state, weak random vortical disturbances are added inside the boundary layer. They are Gaussian vortex rings of random phases and amplitudes, as in previous studies of subsonic [37] and supersonic [38, 39] jets. Their amplitude is tuned in order to yield maximum turbulence intensities of $3 \%$ at the nozzle exit so that the shear layers are initially in a weakly disturbed state.

\section{B. Numerical methodology}

The computation is a well-resolved large-eddy simulation (LES) carried out by solving the filtered compressible Navier-Stokes equations in cylindrical coordinates $(r, \theta, z)$ using high-order, low-dissipation and low-dispersion finitedifference schemes. The spatial derivatives are evaluated using centered eleven-point, fourth-order finite differences [40], and time-integration is performed using a second-order, six-stage Runge-Kutta algorithm. At the end of each time step, grid-to-grid oscillations are removed by the application of a sixth-order selective filter. This filter also serves as an implicit subgrid-scale model used to dissipate subgrid-scale energy near the grid cut-off wavenumber [41, 42]. 
Since the present flow is supersonic, shocks will be formed in and near the jet flow, leading to Gibbs oscillations which are damped using a shock capturing method based on a dilatation-based adaptive filtering [43]. Near the jet axis, the singularity is treated using the method of Mohseni \& Colonius [44] and derivatives in the azimuthal direction are computed using fewer points than permitted by the grid in order to reduce the time-step constraint due to the stability condition [45]. Finally, non-reflecting boundary conditions [46, 47] are applied at the inflow, outflow and radial limits of the computational domain.

\section{Computational parameters}

The computational domain extends down to $60 r_{0}$ in the axial direction, excluding the downstream sponge zone, and out to $18 r_{0}$ in the radial direction and contains a total number of $n_{r} \times n_{\theta} \times n_{z}=526 \times 256 \times 2294=309$ million points. The axial mesh spacing $\Delta z$ is constant and equal to $0.025 r_{0}$ from the nozzle exit at $z=0$ and down to $z=30 r_{0}$, after which it gradually increases and reaches $\Delta z=0.05 r_{0}$ at $z=60 r_{0}$. The radial mesh spacing $\Delta r$ is equal to $0.025 r_{0}$ on the jet axis and reaches a minimum of $\Delta r=0.00625 r_{0}$ at $r=r_{0}$ in order to capture the fine-scale turbulent structures that are formed near the nozzle. Further away from the jet flow, it increases and reaches the value of $\Delta r=0.05 r_{0}$ at $r=5 r_{0}$ after which it remains constant, yielding a cut-off Strouhal number of 5 for an acoustic wave discretized by four points per wavelength. After an initial transient period, the flow and sound fields are recorded during a total time of $2,000 r_{0} / u_{j}$. In particular, the data are collected every $\Delta t=0.1 r_{0} / u_{j}$ on a conical suface spreading with the jet at angle of 11 degrees. The radial location of this surface, represented in Fig. 1. is between $r=r_{0}$ at $z=0$ and $r=7 r_{0}$ at $z=30 r_{0}$. It is thus very close to the turbulent flow in order to study the mechanisms leading to the steepening of Mach waves inside the jet. In addition, the azimuthal Fourier coefficient of the pressure and velocity fields are computed every $\Delta t=0.2 r_{0} / u_{j}$ up to the mode 4 for $0 \leq z \leq 60 r_{0}$ and $0 \leq r \leq 15 r_{0}$. On the whole, approximately 300000 time steps were performed which consumed $80000 \mathrm{CPU}$ hours and generated $2 \mathrm{~TB}$ of data.

\section{Results}

\section{A. Properties of the near acoustic field}

An instantaneous representation of the jet flow and sound fields is provided in Fig. 11, where an isosurface of q-criterion colored by the jet velocity is represented inside the jet flow, and the pressure fluctuations $p^{\prime}=p-p_{\infty}$ are represented outside. In the near pressure field, straight, inclined acoustic waves are present near the jet and propagate with an angle of approximately 45 degrees with respect to the flow direction. They are Mach waves produced by the supersonic motion of large-scale coherent structures inside the jet flow. At certain locations, as at $(r, z)=\left(-3 r_{0}, 7 r_{0}\right)$, for instance, the pressure levels due to these Mach waves are particularly high and the edges of the wavefronts display steep variations suggesting the presence of shocks.

The axial variations of the pressure levels on the surface defined in section II.C are represented in Fig. 2(a). The 

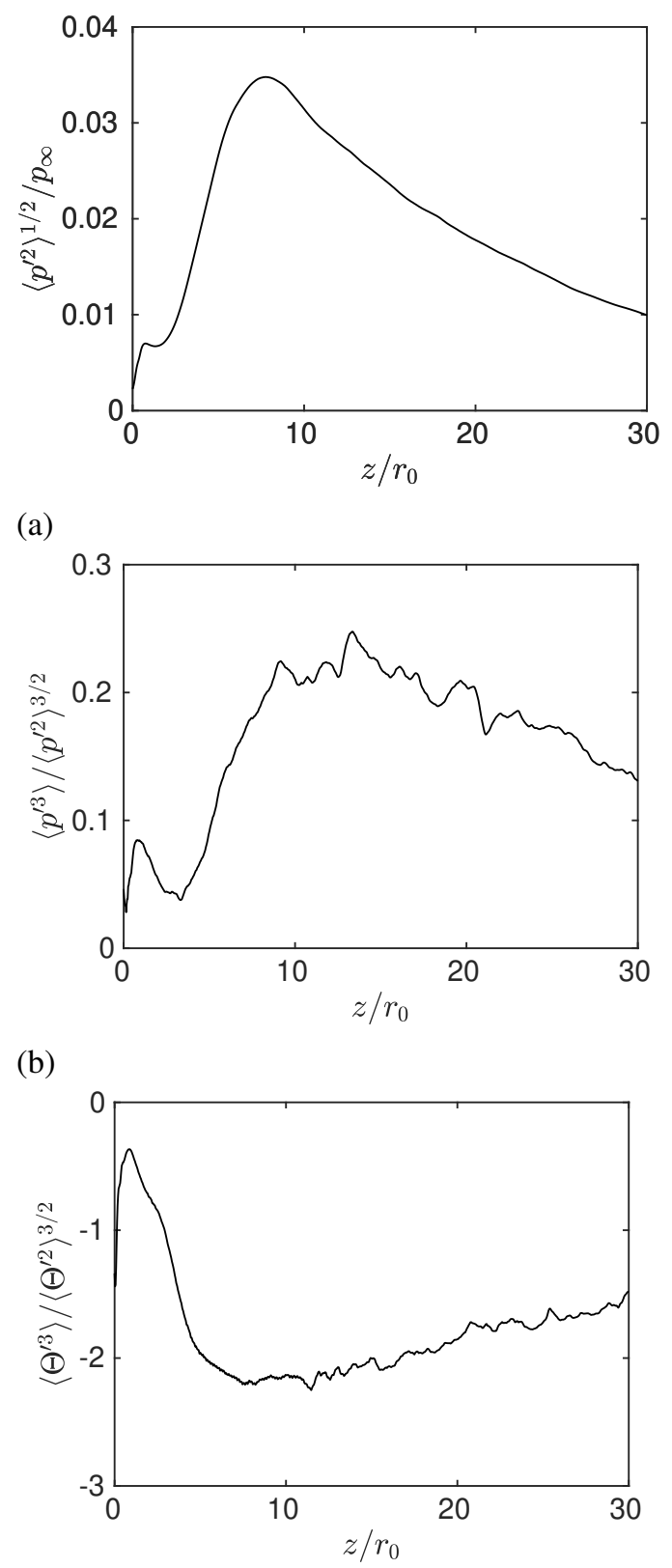

(c)

Fig. 2 Axial variations of the (a) pressure levels (b) pressure skewness and (c) dilatation skewness on the conical surface

levels are low near the nozzle, for $0 \leq z \leq 5 r_{0}$, due to the fact that the mixing layers of the jet are not in a fully-developed turbulent state close to the nozzle exit. Further downstream, the pressure levels rapidly increase and reach a peak of $3500 \mathrm{~Pa}$ at $z \simeq 8 r_{0}$ after which they gradually decrease with the axial distance. The skewness factors of the fluctuations of pressure and of dilatation $\Theta=\nabla \cdot \mathbf{u}$ are represented in Fig. 2 (b,c). For $z \geq 8 r_{0}$, the skewness factors significantly deviate from the value 0 expected for Gaussian signals, indicating that the acoustic waves measured at these locations present a certain degree of nonlinearity consistent with the high pressure levels. The positive skewness of the pressure 
implies that the maxima in the signals are stronger than the minima, while the negative skewness of the dilatation reveals the presence of steep compressions followed by gradual expansions. Such features are characteristic of the steepened waves observed in the pressure signals recorded in the near [4, 16] and far [1, 8, 10] acoustic fields of jets emitting crackle noise.

The power spectrum density of the pressure fluctuations obtained at the location of peak levels, at $z=8 r_{0}$, is shown in Fig. 3 (a). It is broadband and centered around a peak Strouhal number $S t=f D / u_{j} \simeq 0.6$, where $f$ is the frequency. It also has a narrow shape similar to that of the acoustic spectra in the far fields of supersonic jets radiating Mach waves [3]. An example of the pressure signals recorded at the same location is shown in Fig. 33(b). In this signal, two intense peaks, which are higher than three times the standard deviation, are visible at $t=690 r_{0} / a_{\infty}$. These peaks are preceded by a fast compression phase and followed by a gradual expansion and are thus very similar to the shock-like structures observed in the far acoustic fields of supersonic jets [3, 8, 10]. Although the pressure levels at this location are sufficiently high that the waveforms will steepen as they propagate due to nonlinear effects, the close proximity of the conical surface with the turbulent flow indicates that the distinctive, steepened shape of the waves is due, to a large extent, to the wave generation process. More particularly, the strong intermittency of the waves suggests that they are the result of intense intermittent events occuring inside the jet flow.

\section{B. Conditional averaging procedure}

In order to identify and characterize the generation mechanisms of the steepened Mach waves close to the jets, a conditional-averaging procedure is applied. In this approach, extreme pressure peaks are extracted from the signals recorded on the conical surface and used in order to synchronize the flow and sound fields. Then, an ensemble average is performed so that the random, uncorrelated part of the signal is filtered out, revealing the coherent features of the flow correlated with the trigger peaks. This approach has been recently applied by the authors to describe the generation of steepened Mach waves near temporally-developing jets [29-31, 34]. Moreover, similar approaches have also been used to study noise generation mechanisms [49, 50] and to identify and classify coherent structures in high-speed jets [48, 51, 52].

The pressure peaks are extracted by splitting the signals recorded at a given position in intervals of constant length $\tau$. For each intervals, the maximum of the pressure fluctuations is determined over the entire circumference of the jet and their times $t_{\text {trig }}$ and azimuthal positions $\theta_{\text {trig }}$ are stored. The normalized probability density functions (PDF) of the pressure peaks at $z_{\text {trig }}=8 r_{0}$ and $r_{\text {trig }}=2.75 r_{0}$, at the location of peak levels, are shown in Fig. 4 for different values of the interval length $\tau$ ranging from $0.5 r_{0} / a_{\infty}$ to $2 r_{0} / a_{\infty}$. The distributions are not symmetric about their peak, as expected for the PDF of the maxima of random variables. When $\tau$ is increased, the maximum of the PDF is shifted towards higher values so that the intervals have stronger peaks. For instance, $28 \%, 50 \%$ and $78 \%$ of the pressure peaks are higher than $3 p_{r m s}$ for $T=0.5 r_{0} / a_{\infty}, r_{0} / a_{\infty}$ and $2 r_{0} / a_{\infty}$, respectively. Since one objective of the present study is to 


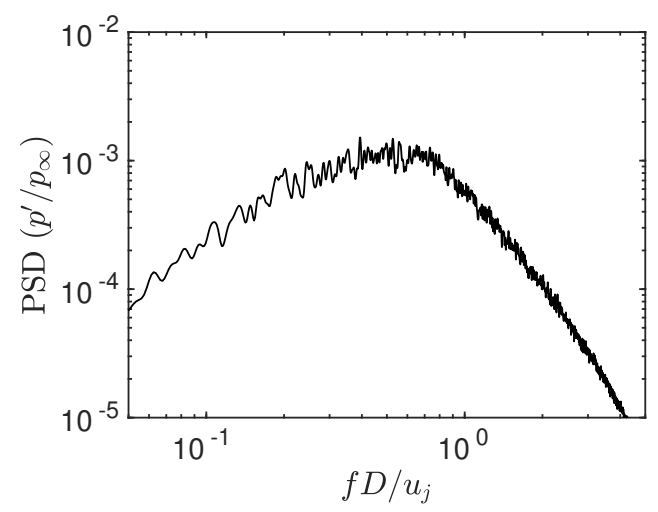

(a)

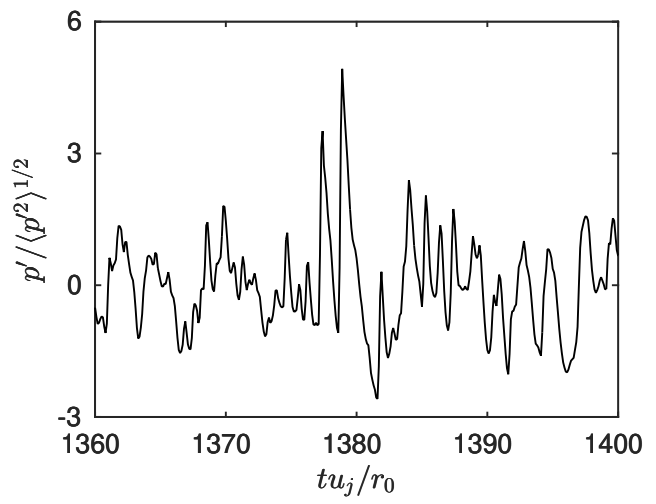

(b)

Fig. 3 Representation at $z=8 r_{0}$ and $r=2.8 r_{0}$ of the (a) power spectrum density normalized by $p_{\infty}$ and (b) time variations of the pressure fluctuations

compare the flow structures correlated with moderate and high-intensity pressure peaks, the value $\tau=r_{0} / a_{\infty}$ is used in what follows as it allows us to extract a wide spectrum of peak amplitudes, ranging from values lower than $2.5 p_{\text {rms }}$ to values higher than $4 p_{r m s}$. Moreover, this time scale corresponds to a Strouhal number of 1 which is close to the peak of the spectra in Fig. 3 (a). The detection procedure is performed at 10 different trigger positions $z_{\text {trig }}$ evenly distributed every $r_{0}$ on the conical surface, ranging from $z=6 r_{0}$, where the pressure skewness becomes significant in Fig. 2 $2 \mathrm{~b}$ ), down to the end of the potential core at $z=15 r_{0}$.

Prior to computing the averages, the extracted peaks are distributed into five groups according to their magnitudes. The first group contains the peaks whose amplitude $p_{\max }$ is lower than $2.5 p_{r m s}$ while the others contains the peaks such that $2.5 p_{r m s} \leq p_{\max }<3 p_{r m s}, 3 p_{r m s} \leq p_{\max }<3.5 p_{r m s}, 3.5 p_{r m s} \leq p_{\max }<4 p_{r m s}$ and $4 p_{r m s} \geq p_{\max }$. For $z_{\text {trig }}=8 r_{0}$, for instance, at the location of peak pressure levels, these groups contain 136, 266, 232, 127 and 40 events, respectively. The conditionally-averaged pressure signals at this location are plotted in Fig. 55(a). The signals obtained for the five groups are very similar and consist of a fast compression phase for $t \leq t_{\text {trig }}$ followed by a gradual expansion phase for $t>t_{\text {trig }}$. Thus, they possess the main features of the steepened waves associated with crackle. It can also be noted that the present structures are very similar to the ones extracted by Baars \& Tinney [3], who applied a shock 


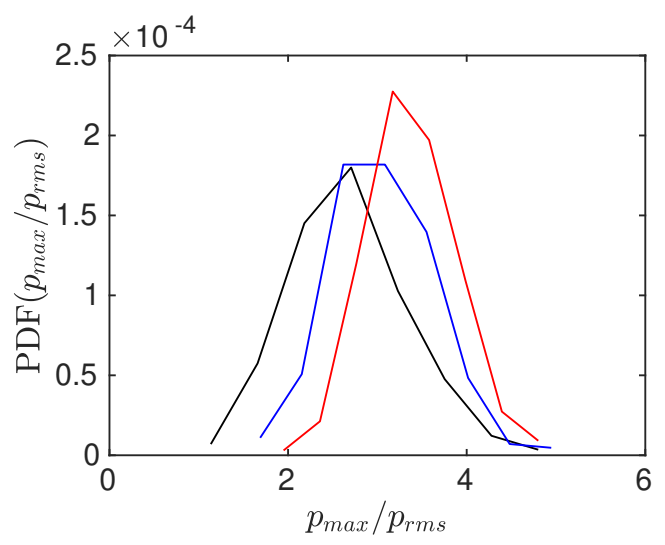

Fig. 4 PDF of the pressure peaks at $z=8 r_{0}$ and $r=2.8 r_{0}$ for interval length $\tau$ of $r_{0} / a_{\infty}$ and $2 r_{0} / a_{\infty}$

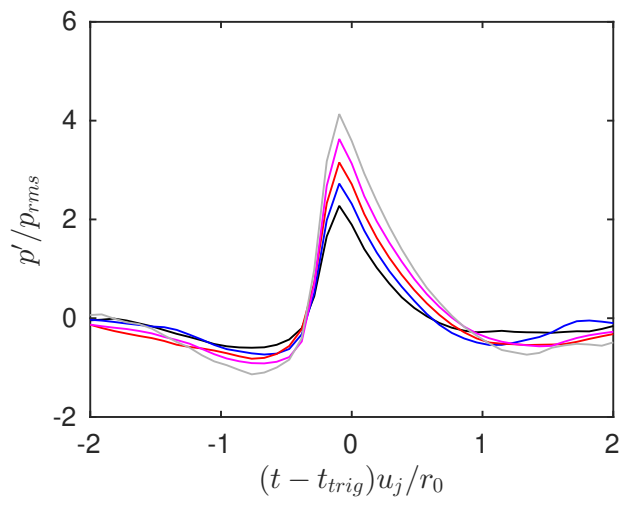

(a)

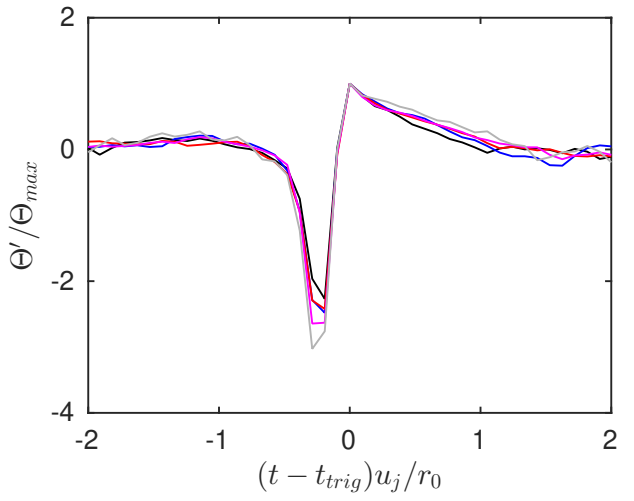

(b)

Fig. 5 Time variations of conditional (a) pressure and (b) dilatation at $z_{\text {trig }}=8 r_{0}$ and $r_{\text {trig }}=2.8 r_{0}$ for $\longrightarrow p_{\max }<2.5 p_{\text {rms }},-2.5 p_{\text {rms }} \leq p_{\max }<3 p_{\text {rms }},-3 p_{\text {rms }} \leq p_{\max }<3.5 p_{\text {rms }}$, $3.5 p_{r m s} \leq p_{\max }<4 p_{r m s}$ and $\quad p_{\max } \geq 4 p_{r m s}$

detection algorithm to pressure signals recorded in the far acoustic field of a cold Mach 3 jet. Since the present analysis is performed in the very near vicinity of the jet flow, this suggests that the distinctive, steepened shapes of the structures already exits at the time when they are generated inside the turbulent flow. 


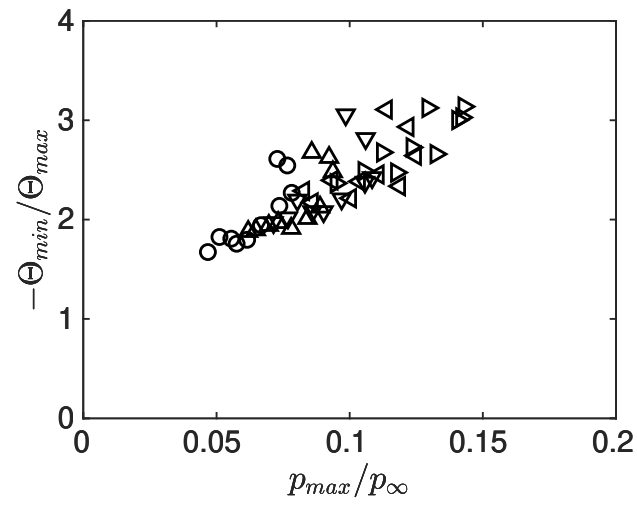

Fig. 6 Conditional steepening factor as a function of the conditional pressure peak for $6 r_{0} \leq z_{\text {trig }} \leq 15 r_{0}$ and $\circ p_{\max }<2.5 p_{r m s}, \triangle 2.5 p_{r m s} \leq p_{\max }<3 p_{r m s}, \nabla 3 p_{r m s} \leq p_{\max }<3.5 p_{r m s}, \triangleleft 3.5 p_{r m s} \leq p_{\max }<4 p_{r m s}$ and $\triangleright$ $p_{\max } \geq 4 p_{\text {rms }}$

The conditional dilatation signals are represented in Fig. 5 (b). They are normalized by their maxima in order to illustrate how the shapes of the waveforms vary with the magnitude of the trigger pressure peaks. In these signals, a marked negative spike due to the steep compression phase is followed by a wide, low-amplitude positive peak related to the expansion phase. The ratio between the minimum and the maximum dilatations increases with the magnitude of the pressure peak, in absolute value. This shows that the slope of the compression phase is more pronounced with respect to that of the expansion phase when the wave amplitude is higher, suggesting a stronger nonlinear behaviour.

A conditional steepening factor $(\mathrm{CSF})$ defined as $\mathrm{CSF}=-\Theta_{\min } / \Theta_{\max }$ is introduced, where $\Theta_{\min }$ and $\Theta_{\max }$ are the minimum and maximum values of the conditional dilatation. It can be viewed as a conditional version of the wave steepening factor introduced by Gallagher \& McLaughlin [9], used in Refs. [3, 10] to assess the nonlinearity of the acoustic waves radiated by supersonic jets. The CSF measures the degree of asymmetry between the compression and expansion phases in a given waveform. While CSF $=1$ corresponds to the case of a smooth, harmonic wave, values higher than one indicate steepened waves. The conditional steepening factor computed on the conical surface for $6 r_{0} \leq z_{\text {trig }} \leq 15 r_{0}$, at the locations indicated as black circles in Fig. 1. and for the five sets of pressure peaks is plotted in Fig. 6 as a function of the conditional pressure peak. It seems to increase linearly so that the highest conditional steepening factors can be associated with the most intense pressure peaks. It can also be noted that all of the CSF values are significantly higher than one, indicating that steepened waves are detected for all of the axial positions considered, which span a large extent of the developing jet shear layer

\section{Coherent structures correlated with the steepened waves}

The conditional averaging procedure is now used to describe the flow events correlated with the steepened Mach waves with the aim to identify their generation mechanisms. For that, the fields are synchronized with respect to the time $t_{\text {trig }}$ and azimuthal coordinate $\theta_{\text {trig }}$ of the pressure peaks, and then averaged. Due to the prohibitive memory cost of 

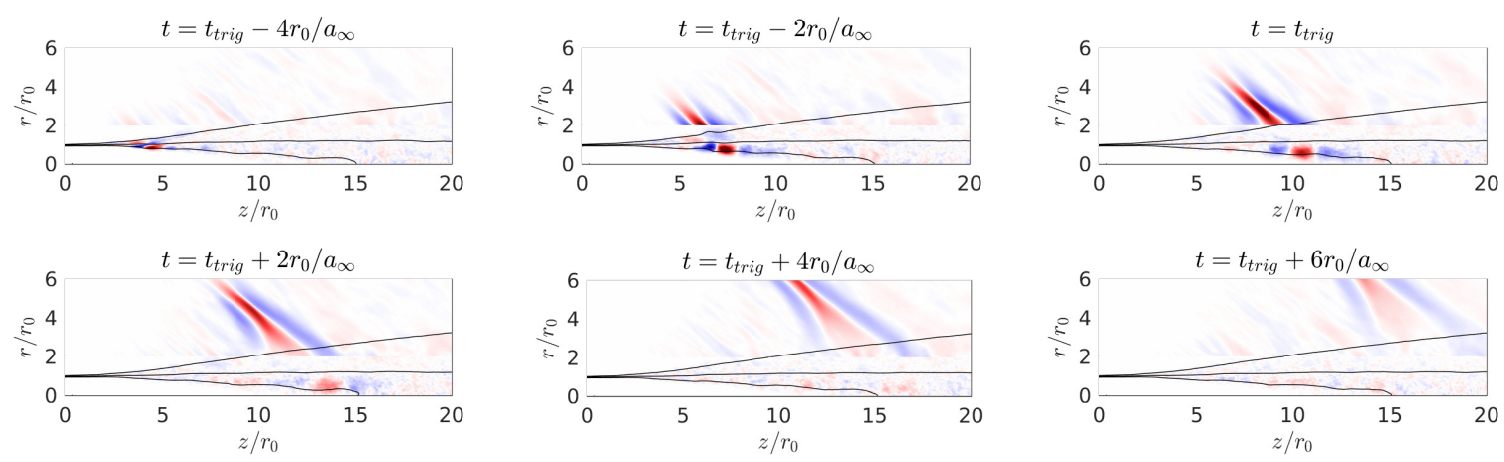

Fig. 7 Representation of conditional pressure fluctuations outside the jet and of conditional vorticity norm inside. The scales range between $\pm 5,000 \mathrm{~Pa}$ for the pressure and $\pm u_{j} / r_{0}$ for the vorticity. The solid lines indicate contours of conditional axial velocity for the values $0.95 u_{j}, a_{\infty}=0.5 u_{j}$ and $0.05 u_{j}$.

storing thousands of three-dimensional arrays of data, the flow and sound fields of the jet are reconstructed using their first five azimuthal Fourier modes before the ensemble average operation. This is justified based on the observation in Ref. [29] that using the first 5 azimuthal modes is sufficient to recover the main features of the steepened waves radiated by the shear layers of supersonic jets.

The conditional fields of vorticity and pressure fluctuations obtained for $z_{\text {trig }}=8 r_{0}$ are represented in Fig. 7 at 6 times between $t_{\text {trig }}-4 r_{0} / a_{\infty}$ and $t_{\text {trig }}+6 r_{0} / a_{\infty}$. In this figure, the conditional fields are obtained using all pressure peaks, so that a total of 801 events are used. The mean vorticity is subtracted in order to remove the component due to the mean shear. The structure of the jet is also represented by showing isocontours of the conditional axial velocity for the values $u_{z}=0.95 u_{j}, a_{\infty}$ and $0.05 u_{j}$, which represent the limit of the potential core, the sonic line and the outer edge of the jet, respectively. In the pressure field, the conditional waveform possesses a distinctive wavepacket structure with an inclination angle of approximately 45 degrees with respect to the jet direction. It is attached to the jet flow at times preceding the trigger $t_{\text {trig }}$ and propagates away for $t>t_{\text {trig. }}$. Inside the jet, the wave is connected to a large-scale vorticity excess located beneath the sonic line and surrounded by two small spots of vorticity deficit. This flow pattern is convected downstream with the wave and can be interpreted as the footprint of the coherent structures in the shear layers. More particularly, the vorticity excess is the trace of large-scale vortices while the vorticity deficits correspond to the stagnation points upstream and downstream of the vortex. When convected at a supersonic speed, these structures act upon the ambient medium in a way similar to that of a supersonic travelling wavy surface, leading to the generation of Mach waves. As argued by Buchta \& Freund [28], the waves generated by such wavy-wall disturbances can steepen due to nonlinear gas dynamics effects when the intensity of the perturbation is strong enough or when their convection speeds are sufficiently high. In the remainder of the paper, the relationship between the intensity of the trigger pressure peaks and properties of the coherent structures, including their convection speed, strength and geometrical shape, are investigated into further details. 


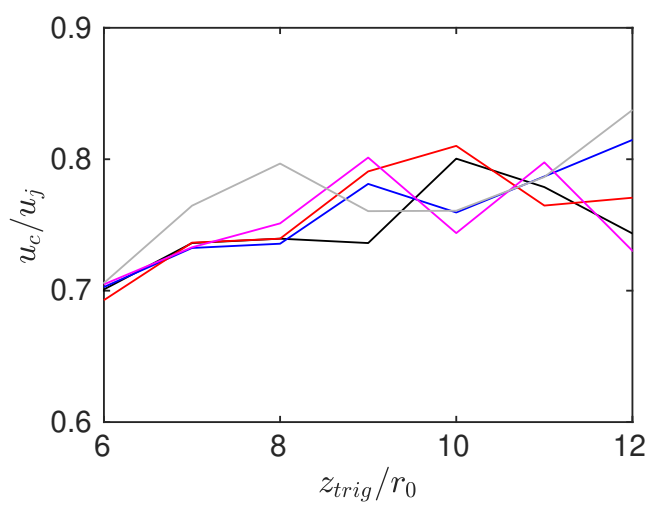

\section{Fig. 8 Axial variations of the convection speed for $-p_{\max }<2.5 p_{\text {rms }},-2.5 p_{\text {rms }} \leq p_{\max }<3 p_{\text {rms }}$, $3 p_{\text {rms }} \leq p_{\max }<3.5 p_{\text {rms }},-3.5 p_{\text {rms }} \leq p_{\max }<4 p_{\text {rms }}$ and $-p_{\max } \geq 4 p_{\text {rms }}$}

\section{Convection speed}

The convection speed of the coherent structures are estimated from the conditional averaged fields by tracking the location of peak vorticity as a function of time and by performing a linear regression. This estimation is carried out separately for the five groups of events in order to identify a possible link between the intensity of the peaks and the convection velocity of the structures. The convection speeds for the five groups are represented in Fig. 8 as a function of the trigger position $z_{\text {trig. }}$. For all groups of events, and for all of the locations considered, it is between $0.7 u_{j}$ and $0.8 u_{j}$, which is in good agreement with the values found in the literature for jets at similar exhaust conditions [20]. It is also significantly higher than the ambient sound speed, and is consistent with the propagation angle of 45 degrees, according to Eq. (1). The convection speed estimated from the conditional averages triggered by the most intense pressure peaks does not appreciably differ from that triggered from the less intense ones. Thus, there is no clear link between the magnitude of the pressure peaks and the convection speed of the coherent structures in the present jet.

\section{Strength of the coherent structures}

The mean strength of the coherent structures correlated with the steepened waves are characterized, following Zaman \& Hussain [48], by considering the maximum of conditional vorticity. The time-variations of this maximum are plotted in Fig. 9 for the trigger position $z_{\text {trig }}=8 r_{0}$, for the five groups of events. In all cases, the maximum vorticity grows and reaches a peak at $t_{s}=t_{\text {trig }}-r_{\text {trig }} / a_{\infty}$ and rapidly decreases afterwards as the structure decays and vanishes. Moreover, the vorticity level is higher for stronger trigger peaks, indicating that the intensity of the waves increases with the strength of the coherent structures. In order to confirm this, the maximum vorticity at $t=t_{s}$ is plotted in Fig. 10 a) as a function of the pressure peak for different trigger positions $z_{\text {trig }}$ represented as black circles in Fig. 1 1 for the five groups of events. An approximately linear relationship is obtained, which illustrates that Mach wave radiation is essentially a linear phenomenom. The nonlinear behaviour of the waves, represented by the conditional steepening factor in Fig. 10 b) also increases with the strength of the vortical structures. Therefore, the strongest large-scale vortices 


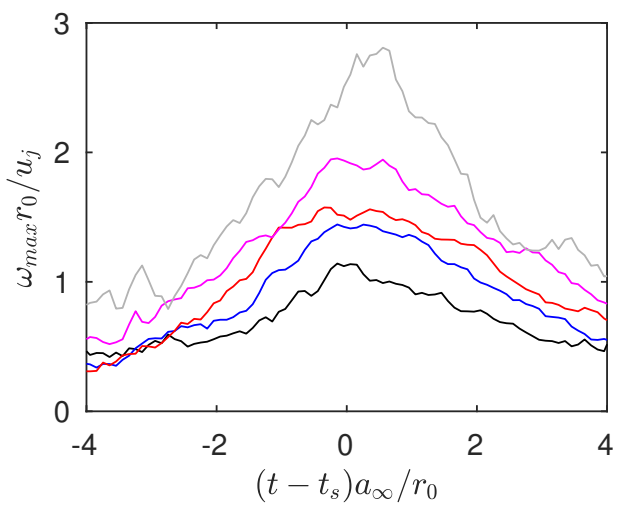

Fig. 9 Maximum conditional vorticity for $z_{\text {trig }}=8 r_{0}$ as a function of $t-t_{s}$, where $t_{s}=t_{\text {trig }}-r_{\text {trig }} / a_{\infty}$ for $-p_{\max }<2.5 p_{\text {rms }},-2.5 p_{\text {rms }} \leq p_{\max }<3 p_{\text {rms }},-3 p_{\text {rms }} \leq p_{\max }<3.5 p_{\text {rms }}$, $3.5 p_{r m s} \leq p_{\max }<4 p_{r m s}$ and $\quad p_{\max } \geq 4 p_{r m s}$

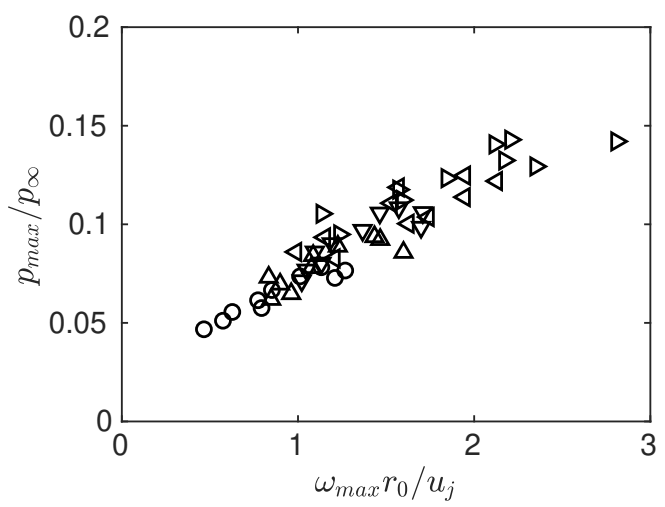

(a)

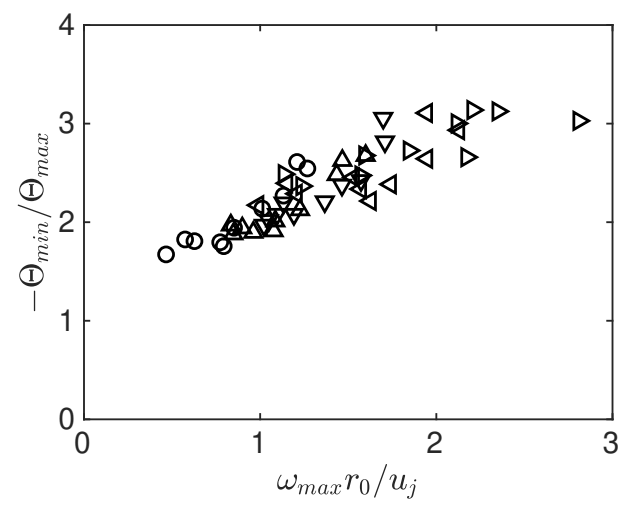

(b)

Fig. 10 Maximum conditional (a) pressure and (b) steepening factor as a function of the peak conditional vorticity for $\circ p_{\max }<2.5 p_{r m s}, \Delta 2.5 p_{r m s} \leq p_{\max }<3 p_{r m s}, \nabla 3 p_{r m s} \leq p_{\max }<3.5 p_{r m s}, \triangleleft 3.5 p_{r m s} \leq p_{\max }<$ $4 p_{r m s}$ and $\triangleright p_{\max } \geq 4 p_{r m s}$

are those that generate the most intense but also the steepest acoustic waves. 


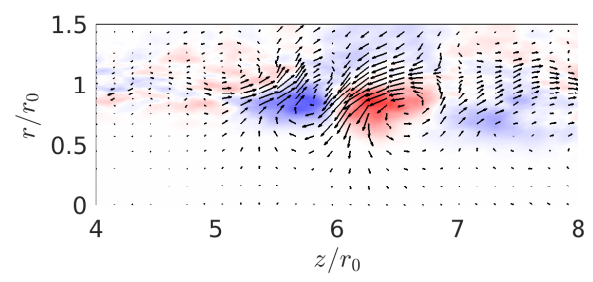

(a)

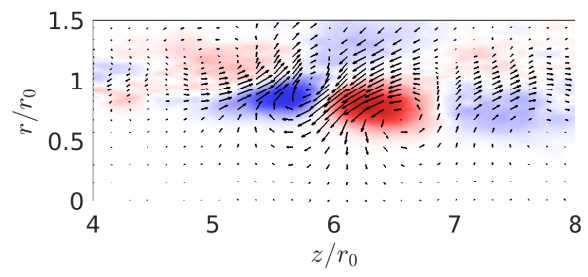

(b)

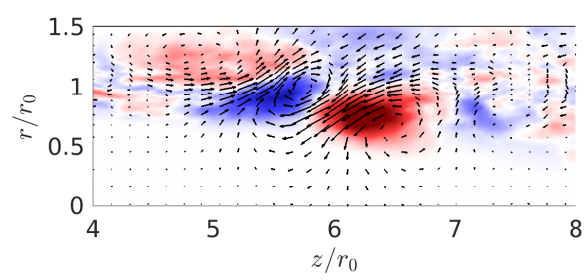

(c)

Fig. 11 Representation at $t=t_{s}$ of the conditional velocity as a vector field with the vorticity in color scale for (a) $p_{\max }<2.5 p_{r m s}$, (b) $3 p_{r m s}<p_{\max } \leq 3.5 p_{r m s}$ and (c) $p_{\max } \geq 4 p_{r m s}$. The scale for the vorticity ranges between $\pm 2 u_{j} / r_{0}$

\section{Geometrical shape of the coherent structures}

Finally, the link between the steepened Mach waves and the geometrical shape of the coherent structures which generate them is explored. For that, the conditional fields of vorticity for $z_{\text {trig }}=8 r_{0}$ are represented in Fig. 11 at $t_{s}=t_{\text {trig }}-r_{\text {trig }} / a_{\infty}$ for three of the five groups of events. The first group contains the weakest events, such that $p_{\max }<2.5 p_{r m s}$, the second one the events of medium intensity, for which $2.5 p_{r m s} \leq p_{\max }<3.5 p_{r m s}$, and the third group the strongest events such that $p_{\max } \geq 4 p_{r m s}$. The conditional velocity field is also represented as a vector field in order to visualize the trace of the coherent structures. The fields in the three cases share the same general features, namely a vorticity excess associated with a large-scale vortex and followed by a vorticity deficit corresponding to the stagnation point downstream from the vortex. The shape of the structures depends on the magnitude of the trigger pressure peak. For the weakest group of events, in Fig.11(a), the vortex and its stagnation point are aligned with respect to the flow direction. However, for the medium-intensity events in Fig. 11(b) and, to a larger extent, for the strongest events in Fig. 11(c), the center of the vortex is located deeper inside the jet flow than the stagnation point, so that the flow disturbance is slightly inclined towards the jet axis with respect to the flow direction. This effect is also visible in 


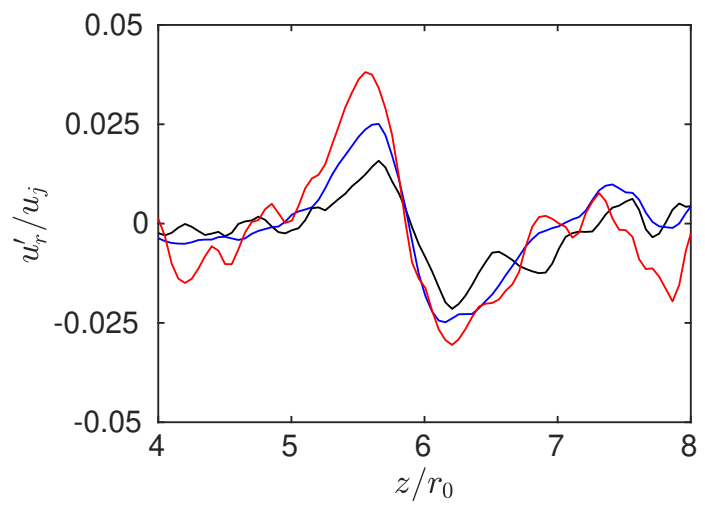

Fig. 12 Profiles of conditional radial velocity fluctutions at $t=t_{s}$ and $r=1.2 r_{0}$ for $-p_{\max }<2.5 p_{r m s}$, $-3 p_{r m s} \leq p_{\max }<3.5 p_{r m s}$ and $-p_{\max } \geq 4 p_{r m s}$

the profiles of conditional radial velocity just above the vortex structure, at $r=1.2 r_{0}$, represented in Fig. 12, For all groups of events, the profiles consist of a peak followed by a trough. The amplitude of the peak is very close to that of the trough for the weakest group of events due to the fact that the vortex center and stagnation point are aligned in the flow direction. For the medium- and high-intensity events, however, the peak is significantly higher than the trough, in absolute value. This asymmetry of the profile can help us explain, at least qualitatively, why the inclined structures generate stronger acoustic waves than the others. Indeed, coherent structures in high-speed jets generate sound by a mechanism analogous to that of a wavy surface convected at a supersonic speed. This wavy wall analogy can help us provide a simplified model of the sound generation mechanisms at play by considering a two-dimensional wall profile based on the variations of the conditional radial velocity in Fig. 12 In a frame of reference moving with the structure at a supersonic convection speed, positive radial velocities, corresponding to positive slopes of the wall, will lead to overpressures while negative velocities will be associated with underpressures. As a consequence, the asymmetric profile obtained for the strongest group of events is likely to generate pressure waveforms with peaks stronger than troughs thus promoting, for a given amplitude of the perturbation, the formation of intense, positively skewed acoustic waves. Despite that the coherent structures depicted above are significantly more complex than a simple wavy wall and that the choice of considering the velocity profile at $r=1.2 r_{0}$ is arbitrary, this provides a simple explanation of the mechanisms by which the geometrical shape of the coherent structures can influence the strength and asymmetry of the Mach waves they generate.

\section{Conclusion}

In the present study, the generation of steepened Mach waves in the near acoustic field of an isothermal Mach 2 jet is investigated by examining a numerical simulation database obtained using large-eddy simulation. The pressure fluctuations recorded on a conical surface located in the near vicinity of the flow and spreading with the jet are found to 
display intense acoustic waves with rapid compressions and gradual expansions. The presence of these waves so close from the turbulent flow indicates that their steepened aspect is due, to a large extent, to nonlinear mechanisms occurring inside the jet. Besides, their strong directivities and large wavelengths suggest that they are a particular, nonlinear case of Mach waves. It is first shown that the steepened aspect of the waves is directly linked to their amplitude, which confirms the nonlinearity of the steepening mechanisms involved. Then, the wave-steepening process is investigated by performing conditional averages triggered by the detection of intense pressure peaks at different axial locations on the conical surface. These averages allow us to establish a direct link between the generation of steepened Mach waves and the supersonic convection of large-scale coherent structures located in the high-speed supersonic core of the jet. The averages are computed separately for different groups of events corresponding to different magnitudes of the trigger pressure peaks. It is first found that the strongest and weakest waves are generated by coherent structures travelling at very similar speeds. Thus, the wide range of wave amplitudes and steepening factors measured near the jet is not due to variations of the convection speed. The strength of the structures, however, plays an important role as the peak of conditional pressure is linearly related to the maximum of conditional vorticity. As a result, the most intense acoustic waves are generated by the strongest coherent structures, which are also those that generate the steepest waves. Finally, the structures correlated with the strongest trigger peaks are more inclined than the others with respect to the flow direction. Following the wavy-wall analogy, the asymmetric shape of these structures causes the generation of positively skewed Mach waves, which favors their steepening through nonlinear effects. Therefore, it appears that the strength and geometrical shapes of the structures are the most important characteristics pertaining to the generation of steepened Mach waves. These results could help us investigate how noise reduction devices such as chevrons, nozzle inserts or microjets act upon the properties of coherent structures, which could be useful in order to optimize noise mitigation strategies.

\section{Acknowledgements}

This work was granted access to the high performance computing (HPC) resources of PMCS2I (Pôle de Modélisation et de Calcul en Sciences de l'Ingénieur et de l'Information) of Ecole Centrale de Lyon, PSMN (Pôle Scientifique de Modélisation Numérique) of ENS de Lyon and P2CHPD (Pôle de Calcul Hautes Performances Dédiés) of Université Lyon I, members of FLMSN (Fédération Lyonnaise de Modélisation et Sciences Numériques), partner of EQUIPEX EQUIP@MESO, and of the resources of IDRIS (Institut du Développement et des Ressources en Informatique Scientifique) under the allocation 2019-2a0204 made by GENCI (Grand Equipement National de Calcul Intensif). It was performed within the framework of the Labex CeLyA of Universite de Lyon, within the programme 'Investissements d'Avenir' (ANR-10-LABX-0060/ANR-16-IDEX-0005) operated by the French National Research Agency (ANR). 


\section{References}

[1] Ffowcs Williams, J. E., Simson, J., and Virchis, V. J., 'Crackle': an annoying component of jet noise, J. Fluid Mech., Vol. 71, pp. 251-271, 1975 doi https://doi.org/10.1017/S0022112075002558.

[2] Laufer, J., Schlinker, R. and Kaplan, R. E., Experiments on supersonic jet noise, AIAA J., Vol. 14, No. 4, pp. 489-497, 1976 doi https://doi.org/10.2514/3.61388

[3] Baars, W. J. and Tinney, C. E., Shock-structures in the acoustic field of a Mach 3 jet with crackle, J. Sound Vib., Vol. 333, pp. 2539-2553, 2014 doi https://doi.org/10.1016/j.jsv.2014.01.008

[4] Mora, P., Heeb, N., Kastner, J., Gutmark, E. J., and Kailasanath, K., Impact of heat on the pressure skewness and kurtosis in supersonic jets, AIAA J., Vol. 52, No. 4, 2014. doi https://doi.org/10.2514/1.J052612

[5] Krothapalli, A., Venkatakrishnan, L., and Lourenco, L., Crackle: A dominant component of supersonic jet mixing noise, AIAA paper 2000-2024, 2000. doi https://doi.org/10.2514/6.2000-2024

[6] Schlinker, R., Liljenberg, S., Polak, D., Post, K., and Chipman, C., Supersonic jet noise source characteristics and propagation: engine and model scale, AIAA paper 2007-3623, 2007. doi https://doi.org/10.2514/6.2007-3623.

[7] Gee, K. L., Sparrow, V. W., James, M. M., Downing, J. M., Hobbs, C. M., Gabrielson, T. B. and Atchley, A. A., The role of nonlinear effects in the propagation of noise from high-power jet aircraft, J. Acoust. Soc. Am., Vol. 123, No. 6, 2008. doi https://doi.org/10.1121/1.2903871

[8] Petitjean, B., Viswanathan, K., and McLaughlin, D., Acoustic pressure waveforms measured in high speed jet noise experiencing nonlinear propagation, Int. J. Aeroacoust., Vol. 5, No. 2, pp. 193-215, 2006. doi https://doi.org/10.1260/2F147547206777629835

[9] Gallagher, J. and McLaughlin, D., Experiments on the nonlinear characteristics of noise propagation from low and moderate Reynolds number supersonic jets, AIAA Paper 1981-2041, 1981. doi https://doi.org/10.2514/6.1981-2041

[10] Fiévet, R., Tinney, C. E., Baars, W. J., and Hamilton, M. F., Coalescence in the sound field of a laboratory-scale supersonic jet, AIAA J., Vol. 54, No. 1, pp. 254-265, 2016. doi:https://doi.org/10.2514/1.J054252

[11] Lighthill, J., The inaugural Theodorsen lecture : some aspects of the aeroacoustics of high-speed jets, Theor. Comp. Fluid. Dyn., Vol. 6, No. 5, pp. 261-280, 1994. doi https://doi.org/10.1007/BF00311841

[12] Hamilton, M. F. and Blackstock, D. T., Nonlinear Acoustics, Chapter 13, p. 383 Acoustical Society of America, 1997.

[13] Baars, W., Tinney, C. E., Wochner, M. S. and Hamilton, M. F., On cumulative nonlinear acoustic waveform distorsions from high-speed jets, J. Fluid Mech., Vol. 749, pp. 331-366, 2014. doi/https://doi.org/10.1017/jfm.2014.228

[14] Papamoschou, D., Evidence of shocklets in a counterflow supersonic shear layer, Phys. Fluids, Vol. 7, No. 2, 233, 1995, pp. 2833-2842. doi:https://doi.org/10.1063/1.868621 
[15] Rossmann, T., Mungal, M. G. and Hanson, R. K., Evolution and growth of large-scale structures in high compressibility mixing layers, J. Turbul., Vol. 3, N9, pp. 2833-2842, 2002. doi https://doi.org/10.1088/1468-5248/3/1/009

[16] Nichols, J. W., Lele, S. K., Ham, F. E., Martens, S., and Spyropoulos, J. T., Crackle noise in heated supersonic jets, J. Eng. Gas Turb. Power, Vol. 135, No. 5, 2013. doi http://dx.doi.org/10.1115/1.4007867

[17] Buchta, D. A., and Freund, J. B., The near-field pressure radiated by planar high-speed free-shear-flow turbulence, J. Fluid Mech., Vol. 832, p. 383-408, 2017. doi 10.1017/jfm.2017.671

[18] Freund, J. B., Lele, S. K. and Moin, P., Numerical simulation of a Mach 1.92 turbulent jet and its sound field, AIAA J., Vol. 38, No. 11, pp. 2023-2031, 2000. doi https://doi.org/10.2514/2.889

[19] Tam, C. K. W., Supersonic jet noise, Annu. Rev. Fluid. Mech., Vol. 27, pp. 17-43, 1995. doi https://doi.org/10.1146/annurev.fl.27.010195.000313

[20] Troutt, T. R. and McLaughlin, D. K., Experiments on the flow and acoustic properties of a moderate-Reynolds-number supersonic jet, J. Fluid Mech., Vol. 116, pp. 123-156, 1982. doi https://doi.org/10.1017/S0022112082000408

[21] Tam, C. K. W., and Burton, D. E., Sound generated by instability waves of supersonic flows. Part 1. Two-dimensional mixing layers, J. Fluid Mech., Vol. 138, pp. 249-271, 1984. doi https://doi.org/10.1017/S0022112084000112.

[22] Tam, C. K. W., and Burton, D. E., Sound generated by instability waves of supersonic flows. Part 2. Axisymmetric jets, J. Fluid Mech., Vol. 138, pp. 273-295, 1984. doi https://doi.org/10.1017/S0022112084000124.

[23] Tam, C. K. W., and Chen, P., Turbulent mixing noise from supersonic jets, AIAA J., Vol. 32, No. 9, pp. $1774-1780$, 1994. doi https://doi.org/10.2514/3.12173.

[24] Sinha, A., Rodriguez, D., Brès, G., and Colonius, T., Wavepacket models for supersonic jet noise, J. Fluid Mech., Vol. 742, pp. 71-95, 2014. doi https://doi.org/10.1017/jfm.2013.660

[25] Martens, S., Spyropoulos, J. T., and Nagel, Z., The effects of chevrons on crackle-engine and scale model results, Proceedings of the ASME Turbo Expo, GT2011-46417, 2011.

[26] Krothapalli, A., Greska, B. and Arakeri, V., High speed jet noise reduction using microjets, AIAA Paper $2002-2450,2002$. doi https://doi.org/10.2514/6.2002-2450

[27] Murray, N. E., and Lyons, G. W., On the convection velocity of source events related to supersonic jet crackle, J. Fluid Mech., Vol. 793, pp. 477-503, 2016. doi https://doi.org/10.1017/jfm.2016.127

[28] Buchta, D. A., and Freund, J. B., Intense sound radiation by high-speed flow: Turbulence structure, gas properties, and near-field gas dynamics, Phys. Rev. Fluids, Vol. 4(4), 2019, p.044605. doi 10.1103/PhysRevFluids.4.044605

[29] Pineau, P., and Bogey, C., Steepened Mach waves near supersonic jets: study of azimuthal structure and generation process using conditional averages, J. Fluid Mech., Vol. 880, pp. 594-619, 2019. doi/https://doi.org/10.1017/jfm.2019.729 
[30] Bogey, C. and Pineau, P., Potential-core closing of temporally developing jets at Mach numbers between 0.3 and 2: Scaling and conditional averaging of flow and sound fields, Phys. Rev. Fluids, 124601, pp.1-25, 2019. doi https://doi.org/10.1103/PhysRevFluids.4.124601

[31] Pineau, P., and Bogey, C., Temperature effects on convection speed and steepened waves of temporally developing supersonic jets, AIAA J., Vol. 58(3), pp. 1227-1239, 2020. doi https://doi.org/10.2514/1.J058589

[32] Dimotakis, P. E., Two dimensional shear-layer entrainment, AIAA J., Vol. 24(11), pp. 1791-1796, 1986. doi https://doi.org/10.2514/3.9525

[33] Brown, G. L., The entrainment and large structures in turbulent mixing layers, Proceeding of the 5th Autralasian Conference on Hydraulics and Fluid Mechanics, pp. 352-359, 1974.

[34] Bogey, C., On noise generation in low Reynolds number temporal round jets at a Mach number of 0.9, J. Fluid Mech., Vol. 859, pp. 1022-1056, 2019. doi https://doi.org/10.1017/jfm.2018.864

[35] Avital, E. J., Musafir, R. E. and Korakianitis, T., Nonlinear propagation of sound emitted by high speed wave packets, J. Comput. Acoust., Vol. 21, No. 2, 250027, 2013. doi http://dx.doi.org/10.1142/S0218396X12500270

[36] Zaman, K. B. M. Q., Effect of initial condition on subsonic jet noise, AIAA J., Vol. 23(9), pp. 1370-1373, 1985. doi https://doi.org/10.2514/1.J058589

[37] Bogey, C., Bailly, C. and Marsden, O. Large eddy simulation of the flow and acoustic field of a Reynolds number $10^{5}$ subsonic jet with tripped exit boundary layers, Phys. Fluids, Vol. 23, 035104, 2011. doi https://doi.org/10.1063/1.3555634

[38] Gojon, R., and Bogey, C., Numerical study of the flow and the near acoustic fields of an underexpanded round free jet generating two screech tones, Int. J. Aeroacoust., Vol. 16, No. 7-8, pp. 603-625, 2017. doi https://doi.org/10.1177/2F1475472X17727606

[39] Nonomura, T., Nakano, H., Ozawa, Y., Terakado, D., Yamamoto, M., Fujii K. and Oyama, A., Large eddy simulation of acoustic waves generated from a hot supersonic jet, Shock Waves, Vol. 29(8), pp. 1133-1154, 2019. doi:https://doi.org/10.1007/s00193019-00895-2

[40] Bogey, C., and Bailly, C., A family of low dispersive and low dissipative explicit schemes for noise computation, J. Comput Phys., Vol. 194, No. 1, pp. 194-214, 2004. doi https://doi.org/10.1016/j.jcp.2003.09.003

[41] Bogey, C., and Bailly, C., Computation of a high Reynolds number jet and its radiated noise using large-eddy simulation based on explicit filtering, Comput. Fluids, Vol. 35, pp. 1344-1358, 2006. doi https://doi.org/10.1016/j.compfluid.2005.04.008

[42] Bogey, C., de Cacqueray, N., and Bailly, C., Turbulence and energy budget in a self-preserving round jet: direct evaluation using large eddy simulation, J. Fluid Mech., Vol. 627, pp. 139-160, 2009. doi https://doi.org/10.1017/S0022112009005801

[43] Bogey, C., de Cacqueray, N., and Bailly, C., A shock-capturing methodology based on adaptative spatial filtering for high-order non-linear computations, J. Comput. Phys., Vol. 228, pp. 1447-1465, 2009. doi https://doi.org/10.1016/j.jcp.2008.10.042 
[44] Mohseni, K., and Colonius, T., Numerical treatment of polar coordinate singularities, J. Comput. Phys., Vol. 157, No. 10, pp. 3593-3600, 2002. doi https://doi.org/10.1006/jcph.1999.6382

[45] Bogey, C., Marsden, O., and Bailly, C., Finite differences for coarse azimuthal discretization and for reduction of effective resolution near origin of cylindrical flow equations, J. Comput. Phys., Vol. 230, pp. 1134-1146, 2011. doi https://doi.org/10.1016/j.jcp.2010.10.031.

[46] Tam, C. K. W., and Dong, Z., Radiation and outflow boundary conditions for direct computation of acoustic and flow disturbances in a nonuniform mean flow, J. Comput. Acoust., Vol. 4, No. 2, pp. 175-201, 1996. doi https://doi.org/10.1142/S0218396X96000040

[47] Bogey, C. and Bailly, C., Three-dimensional non-reflective boundary conditions for acoustic simulations: far field formulation and validation test cases, Acta Acust., Vol. 88(4), pp. 463-471, 2002.

[48] Zaman, K. B. M. Q and Hussain, A. K. M. F., Natural large-scale structures in the axisymmetric mixing layer, J. Fluid Mech., Vol. 138, pp. 325-351, 1984. doi https://doi.org/10.1017/S0022112084000148.

[49] Pérez-Arroyo, C., Daviller, G., Puigt, G., Airiau C. and Moreau, S., Identification of temporal and spatial signatures of broadband shock-associated noise, Shock Waves, Vol. 29, pp. 117-134, 2019. doi https://doi.org/10.1007/s00193-018-0806-4

[50] Schmidt, O. T. and Schmid, P. J., A conditional space-time POD formalism for intermittent and rare events: example of acoustic bursts in turbulent jets, J. Fluid Mech., Vol. 867, R2, 2019. doi https://doi.org/10.1017/jfm.2019.200

[51] Lau, J. C. and Fisher, M. J., The vortex-street structure of 'turbulent' jets. Part 1, J. Fluid Mech., Vol. 67(2), pp. 299-337, 1975. doi https://doi.org/10.1017/S0022112075000328

[52] Mayo, D. E., Daniel, K., Lowe, T. and Ng. W., Statistical Flow Structures in Heated Supersonic Jets with Offset Temperature Non-Uniformities, AIAA Paper 2019-2708, 2019. doi:https://doi.org/10.2514/6.2019-2708 